\title{
Acknowledgement to Reviewers of Algorithms in 2015
}

\author{
Algorithms Editorial Office \\ Published: 22 January 2016 \\ MDPI AG, Klybeckstrasse 64, CH-4057 Basel, Switzerland; algorithms@mdpi.com
}

The editors of Algorithms would like to express their sincere gratitude to the following reviewers for assessing manuscripts in 2015.

We greatly appreciate the contribution of expert reviewers, which is crucial to the journal's editorial decision-making process. Several steps have been taken in 2015 to thank and acknowledge reviewers. Good, timely reviews are rewarded with a discount off their next MDPI publication. By creating an account on the submission system, reviewers can access details of their past reviews, see the comments of other reviewers, and download a letter of acknowledgement for their records. In addition, MDPI has launched a collaboration with Publons, a website that seeks to publicly acknowledge reviewers on a per journal basis. This is all done, of course, within the constraints of reviewer confidentiality. Feedback from reviewers shows that most see their task as a voluntary and mostly unseen work in service to the scientific community. We are grateful to our reviewers for the contribution they make.

Agüera-Pérez, Agustín
Adamatzky, Andy
Alonso, Pedro
Alonso, Andrés M.
Amoretti, Michele
Amundarain, Aiert
Araujo, Alvaro
Argyros, I.K.
Argyros, Ioannis
Attouchb, H.
Ayaz, Hasan
Baraldi, Andrea
Barreiro, A.
Baskonus, Mehmet
Behl, Ramandeep
Bergadano, Francesco
Bermudez, Concepcion
Berretti, Stefano
Berselli, Giovanni
Bi, Zhuming
Biktasheva, Irina
Bokanowski, Olivier
Bonfante, Guillaume
Bos, Jeremy
Bradley, Damon
Brunsdon, Chris
Catinas, E.

Agüera-Pérez, Agustín

Adamatzky, Andy

Alonso, Pedro

Alonso, Andrés M.

Amoretti, Michele

Amundarain, Aiert

Araujo, Alvaro

Argyros, I.K

Attouchb, H.

Ayaz, Hasan

ndrea

Barreiro, A.

Baskonus, Mehmet

Behl, Ramandeep

Bergadano, Francesco

Bermudez, Concepcion

Berretti, Stefano

Berselli, Giovanni

Bi, Zhuming

Biktasheva, Irina

Bokanowski, Olivier

Bonfante, Guillaume

Bos, Jeremy

Brunsdon, Chris

Catinas, E.

\author{
Kaddoum, Georges \\ Kaklamani, Dimitra \\ Kang, Brent \\ Kannan, S.R. \\ Kelk, Steven \\ Khan, Zaheer \\ Kharel, Rupak \\ Kim, Sang-Jae \\ Klimchick, Alexandr \\ Koivisto, Mikko \\ Korf, Richard \\ Kotsiantis, Sotiris \\ Kowalski, Piotr A. \\ Kressner, Daniel \\ Krizanc, Danny \\ $\mathrm{Ku}$, Cheng-Yu \\ Kundu, Sukhamay \\ Kuo, Jian-long \\ Kuznetsov, Yuri \\ Langone, Rocco \\ Lavery, Richard \\ Lecroq, Thierry \\ Lee, Hyun Ki \\ Lee, Annie \\ Lee, Jiann-Shu \\ Lee, Sang-gon \\ Lee, Carman K.M.
}

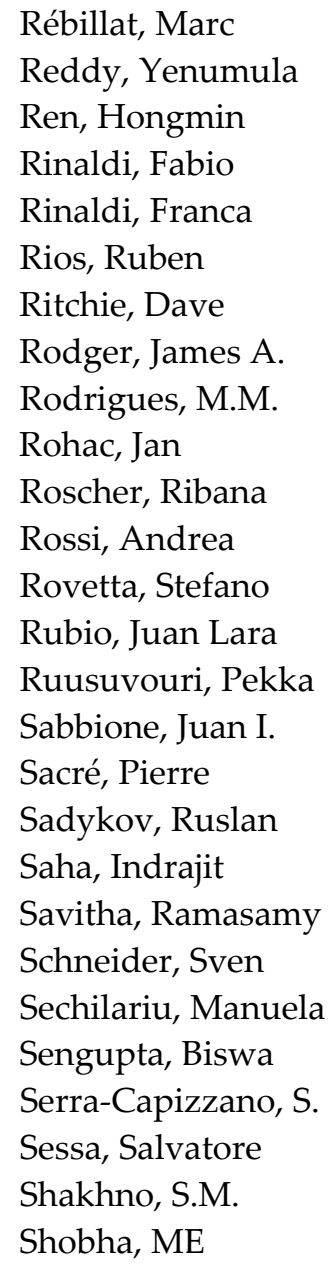

Rébillat, Marc

Reddy, Yenumula

Ren, Hongmin

Rinaldi, Fabio

Rinaldi, Franca

Rios, Ruben

Ritchie, Dave

Rodger, James A.

Rodrigues, M.M.

Rohac, Jan

Rossi, Andrea

Rovetta, Stefan

Ruusuvouri, Pekka

Sabbione, Juan I.

Sacré, Pierre

Sadykov, Ruslan

Saha, Indrajit

Savitha, Ramasamy

Schneider, Sven

Sechilariu, Manuela

Sengupta, Biswa

Serra-Capizzano, S.

Shakhno, S.M.

Shobha, ME 
Cai, Wenli

Cao, Lei

Cassisi, Carmelo

Castro, Eduardo

Chain, Patrick SG

Chan, Keith

Chang, Ruay-Shiung

Chang, Rwei-Ching

Chen, Gang

Chen, Yie-Tone

Chen, Ruey-Maw

Cheng, Jie

Cheng, Chao-Jung

Chervenack, Frank

Chiarandini, Marco

Choi, Hoo-Gon

Chu, Shu-Chuan

Chun, Changbum

Chung, Yao-Liang

Corazza, Marcos

Cordero Fuertes, Juan Antonio

Cormier, G.

Cucala, Lionel

Cuevas, Erik

Czabanski, robert

Dagiuklas, Tasos

Damigos, Matthew

Deane, Charlotte

Densmore, Douglas

Ding, Feng

Diosan, Laura

Dobre, Ciprian

Dondi, Riccardo

Dornaika, Fadi

Dounis, Anastasios I.

Draa, Amer

Du, Jian

Emoto, Kento

Etzlinger, Bernhard

Evgrafov, Anton

Ferrara, Massimiliano

Ferreti, Claudio

Filipovic, Vojislav

Foncubierta Rodríguez, Antonio

Fried, Isaac

Froelich, Wojciech

Gagie, Travis

Gallegos-Funes, Francisco J.

Gansterer, Wilfried N.

Gao, Kaizhou

Gao, Shangce

Garcia, Ander
Lee, Changhoon

Lee, Ming-Chang

Levine, Joshua A.

Li, Jun-Bao

Li, Shuai

Li, Xiang

Li, Jiaming

Li, Lianlin

Lin, Guo-Shiang

Liu, Gang

Liu, Qian

Liu, Yi-Hua

Liu, Hong

Llanes, Antonio

Lukasik, Szymon

Ma, Shiwei

Machado, M.A.S.

Maciel, Carlos Dias

Maciel, Tarcisio Ferreira

Magnenat, Stéphane

Magreñan, A. Alberto

Magreñán, Ángel

Magrenán, Alberto

Maier, Christian

Manlove, David

Marcolin, Federica

Martínez, Eulalia

Mashwani, Wali Khan

Massioni, Paolo

Mavridis, Lazaros

Me, Gianluigi

Meher, Pramod Kumar

Meinel, Christoph

Mena, Hermann

Mendicute, Mikel

Menendez, Hector

Meo, Pasquale De

Miller, Jimmie

Min, Manki

Mirjalili, Seyedali

Misawa, Kazuharu

Moghaddam, Reza Farrahi

Molavi, Behnam

Molina Cantero, Francisco Javier

Moreira, Irina S.

Morrison, Matthew

Moutarde, Fabien

Mújica-Vargas, Dante

Nanni, Loris

Näppi, Janne

Naseer, Noman

Navon, I. Michael
Sivasundaram, Seenith

Soleymani, Fazlollah

Song, Yongzhong

Soundarajan, Sucheta

Spilka, Jiří

Stanco, Filippo

Ştefănescu, R.

Stern, Julio

Suh, Doug Young

Sustik, Mátyás

Suzuki, Hiroyuki

Szabo, Alexandre

Szadkowski, Zbigniew

Szkaliczki, Tibor

Tanweer, M.R.

Tasora, Alessandro

Thulasiraman, $\mathrm{K}$.

Tian, Yun

Timm, Ingo J.

Tizhoosh, Hamid R.

Tomaszuk, Dominik

Traub, Joseph Frederick

Tricco, Terrence

Troncoso, Alicia

Truong-Hong, Linh

Tsai, Yuan-Yu

Tuba, Milan

Turner, Jonathan

Turova, Varvara

Uchizawa, Kei

Uhlmann, Stefan

Ulanov, Alexander

Uschmajew, André

Valenza, Gaetano

Varona, J.L.

Vassileva, María

Vatsalan, Dinusha

Velasco-Forero, Santiago

Venkitasubramaniam, M.

Verbeeck, C.

Verri, M. Cecilia

Vieira, Douglas

Vörös, Jozef

Waldispuhl, Jerome

Walton, S.

Wang, Shitong

Wang, Yu

Wang, He-Sheg

Watel, Dimitri

Wei, Hung-Yu

Chang, Wenlong

Werner, Frank 
Gendron, Paul

Giugno, Rosalba

Gonzalez-Pardo, Antonio

Graffi, Kalman

Gravvanis, George A.

Gross, Jason N.

Gui, Guan

Guimarães, Katia S.

Gunawan, Aldy

Gutiérrez, David

Hajto, Monika

Han, Yahong

Han, Henry

Harbach, Marian

He, Yunlong

He, Zhi

He, Yanyan

Hernandez, Noelia

Hisao, Ishibuchi

Hoang, Duc Chinh

Hon, Cheng-Chuang

Hong, Keum-Shik

Hong, Wien

Horng, Shih-Cheng

Horng, Mong-fong

Houle, Michael E

Hsu, Fang Rong

$\mathrm{Hu}$, Yuanbiao

$\mathrm{Hu}$, Huiyi

Huang, Yuan-Ko

Hwang, J.J.

Jiang, Xue

Jones, Karl O.

Jong, Gwo-Jia
Nersesov, Sergey

Nils, Langenberg

Nonato, Maddalena

Nurunnabi, Abdul

O'Hara, Kieron

Olabarriaga, S.D.

Oliveira, Paulo Moura

Osaba, Eneko

Ouvrard, R

Palazzi, Bernardo

Pandya, Shwetang N.

Papakostas, George A.

Parsopoulos, Konstantinos E.

Patro, Robert

Pedemonte, Martín

Pelta, David A.

Percus, Allon

Pertilä, Pasi

Petriciolet, A.Bonilla

Pham, Son

Pilipchuk, Valery

Piltaver, Rok

Pinho, Armando

Plantié, Michel

Pollonini, Luca

Portmann, Edy

Prakash, Ranganathan

Pulvirenti, Alfredo

Qiu, Jianbin

Quilliot, Alain

Rahman, Ashfaqur

Rao, Yanghui

Rasouli, Mohammad
West, Geoff

Westwick, David

Will, Sebastian

Wojciechowski, Pawel

Wong, Pak-kin

Wong, HK

Xie, Jing

$\mathrm{Xu}$, Qingsong

Xue, Bing

Yamada, Kazunori

Yang, Xin-She

Yang, Chang-Biau

Yanovsky, Igor

Yildiz, Ali R.

Yu, Chenglong

Yu, Han

Yun, Beong In Yun

Yuri, Pirola

Zabalza, Jaime

Zabini, Flavio

Zárate, Luis E.

Zhang, Tianzhu

Zhang, Pengfei

Zhang, Bowu

Zhao, Jun

Zhao, Fang

Zhao, Minbo

Zheng, Zizhan

Zheng, Yufeng

Zhou, Huiyu

Zhou, Qian-Yi

Zhu, Lingli

Zubow, Anatolij

(C) 2016 by the authors; licensee MDPI, Basel, Switzerland. This article is an open access article distributed under the terms and conditions of the Creative Commons by Attribution (CC-BY) license (http://creativecommons.org/licenses/by/4.0/). 